\title{
X-ray line emission from supernova ejecta fragments
}

\begin{abstract}
A. M. Bykov^
A.F. Ioffe Institute for Physics and Technology, St. Petersburg 194021, Russia

Received 4 March 2002 / Accepted 26 April 2002

Abstract. We show that fast moving isolated fragments of a supernova ejecta composed of heavy elements should be sources of $\mathrm{K}_{\alpha} \mathrm{X}$-ray line emission of the SN nuclear-processed products. Supersonic motion of the knots in the intercloud medium will result in a bow-shock/knot-shock structure creation. Fast nonthermal particles accelerated by Fermi mechanism in the MHD collisionless shocks diffuse through a cold metallic knot, suffering from Coulomb losses and producing the X-ray emission. We modeled the X-ray emission from a fast moving knot of mass $M \sim 10^{-3} M_{\odot}$, containing about $\sim 10^{-4} M_{\odot}$ of any metal impurities like Si, S, Ar, Ca, Fe. The accelerated electron distribution was simulated using the kinetic description of charged particles interacting with a strong MHD shock. We accounted for nonlinear effects of shock modification by the nonthermal particle pressure. The $\mathrm{K}_{\alpha}$ line emission is most prominent for the knots propagating through dense molecular clouds. The bow shock should be a radiative wave with a prominent infrared and optical emission. In that case the X-ray line spectrum of an ejecta fragment is dominated by the low ionization states of the ions with the metal line luminosities reaching $L_{\mathrm{x}} \gtrsim 10^{31} \mathrm{erg} \mathrm{s}^{-1}$. High resolution XMM-Newton and Chandra observations are able to detect the $\mathrm{K}_{\alpha}$ and $\mathrm{K}_{\beta}$ line emission from the knots at distances up to a few kpcs. The knots propagating through tenuous interstellar matter are of much fainter surface brightness but long-lived. The line spectra with higher ionization states of the ions are expected in that case. Compact dense knots could be opaque for some X-ray lines and that is important for their abundances interpretation. The ensemble of unresolved knots of galactic supernovae can contribute substantially to the iron line emission observed from the Galactic Center region and the Galactic ridge.
\end{abstract}

Key words. acceleration of particles - radiation mechanisms: non-thermal - ISM: clouds - ISM: individual objects: IC 443 ISM: supernova remnants - X-rays: diffuse background

\section{Introduction}

Physical mechanisms of energy release and nucleosynthesis products ejection in supernova ( $\mathrm{SN})$ events are of fundamental importance for astrophysics. Optical and UV studies of the structure of SN remnants (SNRs) have revealed a complex metal ejecta structure with the presence of isolated highvelocity fragments of SN ejecta interacting with surrounding media. The most prominent manifestations of this phenomena are the fast moving knots (FMKs) observed in some young "oxygen-rich" SNRs in the Galaxy: Cas A (e.g. Chevalier \& Kirshner 1979; Fesen et al. 2002), Puppis A (Winkler \& Kirshner 1985), G292.0+1.8, in the LMC N132D and 1E 0102.2-7219 in the SMC.

Optical FMKs in Cas A are showing very high abundances in O-burning and Si-group elements with high Doppler velocities. They have a broad velocity distribution around $6000 \mathrm{~km} \mathrm{~s}^{-1}$, while bright radio knots are slower (e.g. Bell 1977). The optically emitting mass of the observed knots is only about $10^{-4} M_{\odot}$ (Raymond 1984).

Ejecta-dominated X-ray emission from Cas A was observed with Chandra (Hughes et al. 2000; Hwang et al. 2001) and XMM-Newton (Bleeker et al. 2001; Willingale et al. 2002).

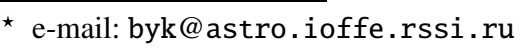

Willingale et al. (2002) found an emission component that is probably due to heating of ejecta clumps sweeping up the ambient gas. They also note that the observed Fe-K line emission is confined to two large clumps. Recent Chandra images of G292.0+1.8 (Park et al. 2001) revealed a complex structure with multiple knots and filaments on angular scales down to the instrumental resolution.

X-ray knots were discovered with ROSAT in the Vela SNR by Aschenbach et al. (1995) as "shrapnel", boomerang structures outside of the main shell. High-resolution Chandra observations of shrapnel A reported by Miyata et al. (2001) revealed a head-tail structure of the apparent size $8.4 \times 44^{\prime} .1(0.6 \times 0.3 \mathrm{pc}$ at $250 \mathrm{pc}$ distance). They estimated the gas pressure in the head to be roughly ten times higher than that in the tail and $T_{\mathrm{e}} \sim$ $0.5 \mathrm{keV}$. The oxygen abundance was $0.34_{-0.08}^{+0.12}$ of the solar value while that of $\mathrm{Si}$ was $3_{-1}^{+2}$ times of solar.

A localized region of $6.4 \mathrm{keV}$ emission indicating the presence of Fe XVII or lower ionization states was found in the supernova remnant RCW 86 with ASCA instrument by Vink et al. (1997).

High resolution Chandra observation of the Galactic Central region reported by Wang et al. (2002) revealed that the observed He-like $\mathrm{Fe} \mathrm{K}_{\alpha}$ emission is largely due to the discrete X-ray source population. Apart from the large-scale thin 
plasma emission in the Galactic Center region Bamba et al. (2002) have also observed with Chandra many small clumps of emission lines from neutral $(6.4 \mathrm{keV})$ to helium-like $(6.7 \mathrm{keV})$, with intermediate line energies between $6.5-6.7 \mathrm{keV}$. The problem of the origin of the observed large scale X-ray emission from the Galactic ridge requires a careful study of possible classes of abundant hard X-ray sources with $L_{\mathrm{x}} \sim 10^{29} \mathrm{erg} \mathrm{s}^{-1}$ (e.g. Tanaka et al. 1999). In this respect modeling of an ensemble of X-ray point sources associated with fast moving SN ejecta fragments seems to be necessary.

In the paper we present a model of X-ray line production in a supersonically moving ejecta fragment. The line emission is due to K-shell ionization by nonthermal particles accelerated by the bow shock and then propagating through a cold metalrich clump. The optical depth effects are important in the model and they are accounted for. Modern arcsec resolution instruments such as that aboard XMM-Newton and Chandra are sensitive to photons up to $10 \mathrm{keV}$ with a good spectral resolution providing unique possibilities to study X-ray line emission of the ejecta fragments. These observations would allow to study stellar nucleosynthesis.

\section{Model}

Consider a fast-moving metal-rich ejecta fragment. Supersonic motion of the fragment (of velocity $v_{\mathrm{k}}$, radius $R$ and mass $M$ ) in the ambient medium will result in a bow-shock/internal-shock structure creation (Chevalier 1975; Sutherland \& Dopita 1995). The bow shock has the velocity $\gtrsim v_{\mathrm{k}}$ and it is propagating in an ambient medium of the standard cosmic composition. The bow shock creates a hot high pressure gas and also accelerates nonthermal particles. The high pressure gas in the head of the fast moving ejecta fragment could drive an internal shock of velocity $v_{\text {is }}$ into the fragment if $v_{\text {is }}$ exceeds the sound speed in the cold matter:

$v_{\mathrm{is}} \approx v_{\mathrm{k}}\left(\rho_{\mathrm{a}} / \rho_{\mathrm{k}}\right)^{1 / 2} \propto v_{\mathrm{k}} \rho_{\mathrm{a}}^{1 / 2} R^{3 / 2} M^{-1 / 2}$.

Here $\rho_{\mathrm{a}}$ and $\rho_{\mathrm{k}}$ are the ambient gas and the dense fragment densities, respectively. We shall also use the density contrast $\chi=\rho_{\mathrm{k}} / \rho_{\mathrm{a}}$. The characteristic size $R$ of $10^{16}-10^{17} \mathrm{~cm}$ is consistent with that resolved by optical observations of Cas A and Puppis A while large clumps were inferred by Chandra observations of Vela shrapnel A by Miyata et al. (2001). For an ejecta fragment of radius $R_{16} \sim 3$ (where $R_{16}$ is the radius measured in $\left.10^{16} \mathrm{~cm}\right)$ and mass $M \sim 10^{-3} M_{\odot}$ the density ratio $\rho_{\mathrm{a}} / \rho_{\mathrm{k}}=\chi^{-1}$ is about $4 \times 10^{-4}$ for an ambient medium of number density $\sim 0.1 \mathrm{~cm}^{-3}$. However the ratio could be as high as $\gtrsim 0.1$ for the ejecta fragments moving through a dense ambient matter of a molecular cloud.

There is a number of factors limiting the fast ejecta fragment lifetime. A fast moving knot is decelerating due to the interaction with the ambient gas. The deceleration time of a knot can be estimated as $\tau_{\mathrm{d}} \approx M v_{\mathrm{k}}^{2} / \rho_{\mathrm{a}} v_{\mathrm{k}}^{3} S \sim \chi R / v_{\mathrm{k}}$, where $S$ is the effective crossection of the bow shock. However, the fast knot hydrodynamical crushing effects could have much shorter timescale, providing $\tau_{\mathrm{c}} \sim \chi^{1 / 2} R / v_{\mathrm{k}} \sim R / v_{\mathrm{is}} \propto$ $M^{1 / 2}\left(R \cdot \rho_{\mathrm{a}}\right)^{-1 / 2} v_{\mathrm{k}}^{-1}($ e.g. Wang $\&$ Chevalier 2001 and references therein). That implies $\tau_{\mathrm{c}} / \tau_{\mathrm{d}} \sim \chi^{-1 / 2} \propto R^{3 / 2} \rho_{\mathrm{a}}^{1 / 2} M^{-1 / 2}$. The knot ablation process with surface erosion could also be a limiting factor.

The hydrodynamical estimation of the inner shock velocity $v_{\text {is }}$ given by Eq. (1) assumes an efficient conversion of the bow shock ram pressure to the knot internal shock. That is a good approximation if the effects of energy loss due to nonthermal particle acceleration and radiative cooling of high pressure gas behind a bow shock are negligible. The latter is the case unless the knot is interacting with dense matter (like molecular cloud clumps).

The Alfvenic Mach number of both the bow shock and the inner shock (if $v_{\text {is }}$ is determined by Eq. (1)) is

$\mathcal{M}_{\mathrm{a}}=v_{\mathrm{k}}\left(4 \pi \rho_{\mathrm{a}}\right)^{1 / 2} / B \approx 460 v_{8} n_{\mathrm{a}}^{1 / 2} / B_{-6}$

where $n_{\mathrm{a}}$ is the ionized ambient gas number density measured in $\mathrm{cm}^{-3}, B_{-6}$ is the local magnetic field just before the shock measured in $\mu \mathrm{G}$ and $v_{8}$ is the flow velocity in $10^{8} \mathrm{~cm} \mathrm{~s}^{-1}$. Note here that the Alfvenic Mach number of the inner shock depends on the ambient density but on the magnetic field inside the knot. The sonic Mach number for a shock propagating in a plasma of the standard abundance is

$\mathcal{M}_{\mathrm{s}} \approx 85 v_{8} \cdot\left[T_{4} \cdot\left(1+f_{\mathrm{ei}}\right)\right]^{-1 / 2}$,

where $T_{4}$ is the plasma ion temperature measured in $10^{4} \mathrm{~K}$ and $f_{\mathrm{ei}}=T_{\mathrm{e}} / T_{\mathrm{i}}$. In general, for the processes in the precursor and viscous velocity jump of an MHD collisionless shock wave no equilibration between electrons and ions should be assumed (e.g. Raymond 2001). Both cases of $f_{\mathrm{ei}} \ll 1$ and $f_{\mathrm{ei}}>1$ could be relevant to the system under consideration. The photoionized gas at the radiative precursor of a fast shock is assumed to have $T_{\mathrm{e}} \sim(1-2) \times 10^{4} \mathrm{~K}$ and $f_{\mathrm{ei}} \gtrsim 1$ (e.g. Shull \& McKee 1979). On the other hand, plasma heating due to MHD waves dissipation in the vicinity of the viscous subshock heats mostly the ions, providing $f_{\mathrm{ei}}<1$.

\subsection{The effect of nonthermal particles on a strong bow shock}

For strong collisionless shocks in a magnetized plasma the nonthermal particle acceleration effect is expected to be efficient and a significant fraction of ram pressure is transferred to high energy particles (ions for nonrelativistic shocks). The shock transition of a strong shock of the total Mach number $\mathcal{M t} \gg$ 1 is broadened because of the upstream gas deceleration by nonthermal particle pressure gradient ahead of the viscous gas subshock of a modest Mach number $\mathcal{M}_{\text {sub }} \leq 3$ (see for a review Blandford \& Eichler 1987; Jones \& Ellison 1991; Malkov \& Drury 2001).

The total compression ratio $R_{\mathrm{t}}$ of a strong MHD shock modified by an efficient nonthermal particle acceleration depends on the energy flux carried out by escaping nonthermal particles and the effective adiabatic exponent (e.g. Malkov \& Drury 2001). On the other hand the distribution function of nonthermal particles and the bulk flow profile in the shock upstream region are sensitive to the total compression ratio $R_{\mathrm{t}}$. Thus, the exact calculation of the escape flux can be performed only in 
fully nonlinear kinetic simulations. Nevertheless, some approximate iterative approach can be used to make the distribution function consistent with the shock compression. The subshock is the standard gas viscous shock with the compression ratio: $R_{\text {sub }}=\left(\gamma_{\mathrm{g}}+1\right) \mathcal{M}_{\text {sub }}^{2} /\left[\left(\gamma_{\mathrm{g}}-1\right) \mathcal{M}_{\text {sub }}^{2}+2\right]$. The downstream ion temperature $T^{(2)}$ behind the modified shock structure can be calculated if $R_{\mathrm{t}}$ and $R_{\text {sub }}$ are known for the shock of the given velocity and it could be very different from that obtained with the standard single-fluid Rankine-Hugoniot law. The electron temperature just downstream of the shock is expected to be lower than $T^{(2)}$.

An exact modeling of the collisionless shock structure with nonthermal particle acceleration effect requires a nonperturbative selfconsistent description of a multi-component and multiscale system including strong MHD turbulence dynamics. That modeling is not feasible at the moment. We shall use some simplified description of a strong shock with some apropriate parameterization of governing parameters which are: $(a)$ the nonthermal particle diffusion coefficients; $(b)$ the ion injection rate; (c) the maximum momentum of accelerated particles. The gas heating mechanism due to MHD waves dissipation in the shock precursor must also be specified in that simplified approach, providing the connection between the total compression and the gas subshock Mach number $\mathcal{M}_{\text {sub }}$. Assuming that the main heating mechanism of the gas in the precursor region is due to Alfven waves dissipation, Berezhko \& Ellison (1999) obtained a simple relation for the total compression of the shock $R_{\mathrm{t}} \approx 1.5 \mathcal{M}_{\mathrm{a}}^{3 / 8}$, while the subshock had $\mathcal{M}_{\text {sub }} \sim 3$. The relation is valid under the condition of $\mathcal{M}_{\mathrm{s}}^{2} \gg \mathcal{M}_{\mathrm{a}}$ in the far upstream flow. They also noted that the subshock compression ratio is not too sensitive to the ion injection rate if the rate exceeds $5 \times 10^{-5}$ to provide the shock modification by the accelerated ions. We obtain then the ion temperature just behind the modified strong shock (measured in $10^{6} \mathrm{~K}$ )

$T_{6}^{(2)} \approx 0.32 \cdot \phi\left(\mathcal{M}_{\mathrm{sub}}\right) \cdot v_{\mathrm{k} 8}^{5 / 4} n_{\mathrm{a}}^{-3 / 8} B_{-6}^{3 / 4}\left(1+f_{\mathrm{ei}}\right)^{-1}$,

where $\phi\left(\mathcal{M}_{\text {sub }}\right)=\left[2 \gamma_{\mathrm{g}} \mathcal{M}_{\text {sub }}^{2}-\left(\gamma_{\mathrm{g}}-1\right)\right] /\left[\left(\gamma_{\mathrm{g}}-1\right) \mathcal{M}_{\mathrm{sub}}^{2}+2\right]$, and $\phi(3) \approx 3.7$ for $\gamma_{\mathrm{g}}=5 / 3$. Then the electron and ion temperatures equilibrate in the postshock region. The initial electron temperature in the shock downstream depends also on the collisionless electron heating (see e.g. Bykov \& Uvarov 1999; Laming 2001). The turbulent gas heating due to acoustic instability wave dissipation could be more efficient however, if the acoustic instability develops (Malkov \& Drury 2001).

The maximal momenta of the accelerated ions are limited by MHD wave damping due to ion-neutral collisions in the case of a fragment interacting with dense molecular cloud. The effect was accounted for following the work by Drury et al. (1996). The maximal momenta of the ions are $\$ 10^{2} \mathrm{GeV} / \mathrm{c}$ in that case. In the case of a fragment propagating through a tenuous medium the maximal momentum is limited by the finite scale of the strong scattering region with typical values of the maximal momentum $\gg 10^{2} \mathrm{GeV} / \mathrm{c}$.

It should be noted that we still have no direct observational evidence for the cosmic ray ions accelerated in SNR shocks. At the same time there is direct evidence for ion and electron acceleration by strong cosmic-ray modified shocks in the interplanetary medium (e.g. Terasawa et al. 1999). In the interplanetary shock of velocity $\sim 375 \mathrm{~km} \mathrm{~s}^{-1}$ (in the solar wind frame) studied by Terasawa et al. (1999) with the GEOTAIL satellite the partial pressure of accelerated nonthermal particles was found to be in a rough balance with the thermal proton pressure in the shock downstream. That implies a high efficiency of nonthermal ion production. The diffusion coefficients for both electrons and ions were estimated to be $\sim 10^{18} \mathrm{~cm}^{2} \mathrm{~s}^{-1}$ in the shock vicinity that is at least two order of magnitude smaller than the typical interplanetary values (Shimada et al. 1999). For SNR shocks Dorfi (2000) estimated the ion acceleration efficiency to be 0.24 for some reasonable parameters.

The observed synchrotron emission from SN shells has been considered for a long time as direct evidence for relativistic electron acceleration. The efficiency of transformation of the kinetic energy of a nonrelativistic shock bulk flow into the nonthermal electron population is not expected to be very high, being below a few percent. However even a percent range efficiency of the nonthermal electron acceleration would provide an observable effect of $\mathrm{K}_{\alpha}$ photon emission from metallic knots irradiated by high energy electrons. Bulk heating and ionization of the knot interior due to deep penetration of energetic ions and electrons could also be important for enhanced ablation.

\subsection{Nonthermal electron acceleration}

To simulate the spectra of nonthermal electrons in the fragment body we solve the kinetic equation for the distribution function of the electrons, accounting for injection, diffusive transport, advection, first and second order Fermi acceleration and synchrotron and the Coulomb losses. Electron kinetics in supercritical collisionless shocks was modeled by Bykov \& Uvarov (1999). They showed that strong MHD fluctuations generated by kinetic instabilities of ions are responsible for heating and pre-acceleration of nonthermal electrons on a very fine scale, of the order of several hundreds of inertial lengths, in the vicinity of the viscous jump (subshock) of a collisionless shock. We have described the details of the code, diffusion model and loss functions used elsewhere (Bykov \& Uvarov 1999; Bykov et al. 2000).

There are three zones in the one-dimensional model: the pre-shock region (I), the shock transition region (II), and the post-shock flow (III), from where nonthermal emission from shock-accelerated particles originates. In order to calculate the spectra of nonthermal electrons in these regions, we used the following kinetic equation for the nearly-isotropic distribution function $N_{i}(z, p)(i=\mathrm{I}-\mathrm{III})$ :

$$
\begin{gathered}
k_{i}(p) \frac{\partial^{2} N_{i}(z, p)}{\partial z^{2}}+\frac{1}{p^{2}} \frac{\partial}{\partial p} p^{2}\left[D_{i}(p) \frac{\partial N_{i}}{\partial p}+L_{i}(p) N_{i}\right] \\
-u_{i}(z) \frac{\partial}{\partial z} N_{i}+\frac{p}{3} \frac{\partial}{\partial p} N_{i}\left[\frac{\partial}{\partial z} u_{i}\right]=0
\end{gathered}
$$

This Fokker-Planck-type equation takes into account diffusion and advection [bulk velocity $u_{i}(z)$ ] of electrons in phase space due to interactions with MHD waves and the large-scale MHD flow. Here $z$ is the coordinate along the shock normal. $L_{i}(p)$ is the momentum loss rate of an electron due to Coulomb 
collisions in a partially ionized plasma and synchrotron/inverse Compton radiation. The momentum diffusion coefficient $D(p)$ is responsible for the second order Fermi acceleration, and $k_{i}(p)$ is the fast particle spatial diffusion coefficient. For lowenergy electrons the Coulomb and ionization losses are important everywhere except for the narrow shock transition region (II), where acceleration is fast enough to overcome the losses and where nonthermal electron injection occurs.

To accelerate the electrons injected in the shock transition region to relativistic energies MHD turbulence should fill the acceleration region. As we know from interplanetary shock observations, turbulence generation is a generic property of collisionless MHD shocks. We used the parameterization of the momentum dependent diffusion coefficient $k_{i}(p)$ of an electron of momentum $p$, described in details by Bykov et al. (2000). The diffusion coefficients are momentum dependent with $k_{i}(p) \propto k_{0} \cdot v p$ above $1 \mathrm{keV}$. The normalization value $k_{0}$ is the electron diffusion coefficient at $1 \mathrm{keV}$.

The electron temperature in the (photo)ionized shock precursor was fixed to $T_{\mathrm{e}}=2 \times 10^{4} \mathrm{~K}$ for the fragments propagating in the dense clouds of the gas of number density $\gg 1 \mathrm{~cm}^{-3}$. The fragment velocity should exceed $\sim 250 \mathrm{~km} \mathrm{~s}^{-1}$ to provide enough $\mathrm{UV}$ radiation to complete $\mathrm{H}$ and $\mathrm{He}\left(\right.$ to $\left.\mathrm{He}^{+}\right)$ionization in the precursor of the bow shock, if it is modified by the nonthermal particle pressure. The velocity is higher than the value of $\sim 110 \mathrm{~km} \mathrm{~s}^{-1}$ derived by Shull \& Mckee (1979), because of the weaker gas heating by the modified MHD shock structure with a smooth precursor and a gas subshock of $\mathcal{M}_{\text {sub }} \sim 3$. It should be noted however that even at lower shock velocities where the upstream photoionization is inefficient, the preshock ionization could be provided by the fast particles accelerated at the collisionless shock. For the fragments propagating in hot tenuous plasma the electron temperature was assumed to be equal to the plasma temperature (i.e. $f_{\mathrm{ei}}=1$ ).

The energy spectrum of the nonthermal electrons in the bow shock of a metal-rich ejecta fragment is shaped by the joint action of the first and second order Fermi acceleration in a turbulent plasma with substantial Coulomb losses. The electron spectra are calculated for different values of the bow shock velocity assuming that the diffusion coefficient is parameterized as in Bykov et al. (2000) with different values of $k_{0}$ for $1 \mathrm{keV}$ electron for different environments. The minimal value of the diffusion coefficient of an electron is $k_{0}^{\min } \approx$ $6.6 \times 10^{16} B_{\mu \mathrm{G}}^{-1}(E / \mathrm{keV}) \mathrm{cm}^{2} \mathrm{~s}^{-1}$. Note that the minimal value is only achievable if strong fluctuations of the magnetic field are present at the electron gyro-radii scale. For slightly superthermal electrons the fluctuations cannot be generated by the ions. Whistler type fluctuations could provide the required electron scale fluctuations (e.g. Levinson 1996). However in the case of a strong MHD turbulence the electron transport by the vortex type fluctuations of ion gyro-radii scale would provide an effective diffusion coefficient much larger than $k_{0}^{\min }$ (Bykov \& Uvarov 1999). We consider here rather a conservative case of that large diffusion coefficient.

The electron acceleration model described above is onedimensional. The transverse gas flow of velocity $u_{\perp}$ behind the bow shock front would affect the nonthermal particle distribution. The exact 2-D and 3-D modeling of the postshock flow with account of shock modification effect is not feasible at the moment. However, the transverse flow effect on high energy electrons is only marginal if the particle diffusion time across the transverse flow is shorter than the transverse advection time scale i.e. $\delta_{\perp} / R \cdot u_{\perp} \delta_{\perp} / k(p)<1$. Here $\delta_{\perp}$ is the width of the transverse gas flow behind the bow shock (typically it is a relatively small fraction of the fragment radius $R$ ). In our case the condition is satisfied for $100 \mathrm{keV}-\mathrm{MeV}$ regime electrons dominating the $\mathrm{K}_{\alpha}$ lines production in the ejecta fragment body. We account for the presence of a postshock cooling layer of the shocked ambient matter situated in front of the metal rich fragment. The curves 1-3 in Fig. 1 illustrate the effect of Coulomb losses on low energy nonthermal electrons diffusing through the postshock layer.

\subsection{Ambient conditions and the fragment state}

As a generic example we consider a fast moving fragment of $v_{\mathrm{k}} \sim 1000 \mathrm{~km} \mathrm{~s}^{-1}$, mass $M=10^{-3} M_{\odot}$ and radius $R_{16}=3$ dominated by oxygen and containing about $\sim 10^{-4} M_{\odot}$ of any metal impurities like $\mathrm{Si}, \mathrm{S}, \mathrm{Ar}, \mathrm{Ca}, \mathrm{Fe}$. That fragment we refer to as "the standard knot".

The electron distribution was simulated here for two distinct representative cases of the ambient environment. The first one is an SN fragment propagating through a dense ambient medium of number density $\sim 10^{3} \mathrm{~cm}^{-3}$, relevant to an SN explosion in the vicinity of a molecular cloud. If the ambient magnetic field follows the scaling $B_{\mathrm{a}} \propto n^{1 / 2}$ (e.g. Hollenbach $\&$ McKee 1989) one may expect the magnetic field values of $\gtrsim 100 \mu \mathrm{G}$ in the dense ambient medium (cf. Blitz et al. 1993). The second case is a $\mathrm{SN}$ fragment propagating through a tenuous medium of number density $\sim 0.1 \mathrm{~cm}^{-3}$, temperature $T \gtrsim$ $10^{4} \mathrm{~K}$ and magnetic field of $\mu \mathrm{G}$ range. Correspondingly, the diffusion coefficient normalization factors for $\mathrm{keV}$ regime electrons were $k_{0} \sim 10^{18} \mathrm{~cm}^{2} \mathrm{~s}^{-1}$ in the dense medium case and $k_{0} \lesssim 10^{20} \mathrm{~cm}^{2} \mathrm{~s}^{-1}$ for the tenuous medium. The gas ionization state in the bow shock downstream was simulated here as described in the paper of Bykov et al. (2000).

The gas ionization state inside an oxygen rich fragment body was estimated following the model by Borkowski \& Shull (1990). They studied the structure of a steady state radiative shock (inner shock) of velocities $v_{\text {is }}$ from 100 to $170 \mathrm{~km} \mathrm{~s}^{-1}$ (cf. Eq. (1)) in a pure oxygen gas with electron thermal conduction. The postshock temperature behind the $140 \mathrm{~km} \mathrm{~s}^{-1}$ shock with thermal conduction was found to be $270000 \mathrm{~K}$ in the immediately postshock region and dropped to roughly $4700 \mathrm{~K}$ after the oxygen depth about $2 \times 10^{15} \mathrm{~cm}^{-2}$ in the downstream photoionization zone. The dominant ionization state of oxygen in the shock radiative precursor and in the immediate postshock region was OIII while it was OII in the downstream photoionization zone. Nonthermal electrons could also be an important factor in modeling the dominant ionization state of the ions in the fragment body (Porquet et al. 2001).

In Fig. 1 the nonthermal parts of the electron distribution functions $\tilde{N}(p, x)=N(p, x) p^{2} p_{\mathrm{T}}$ are shown at the different depths of an SN fragment. Here $p_{\mathrm{T}}$ is the momentum of a thermal electron in the far upstream region. Note that the upstream 


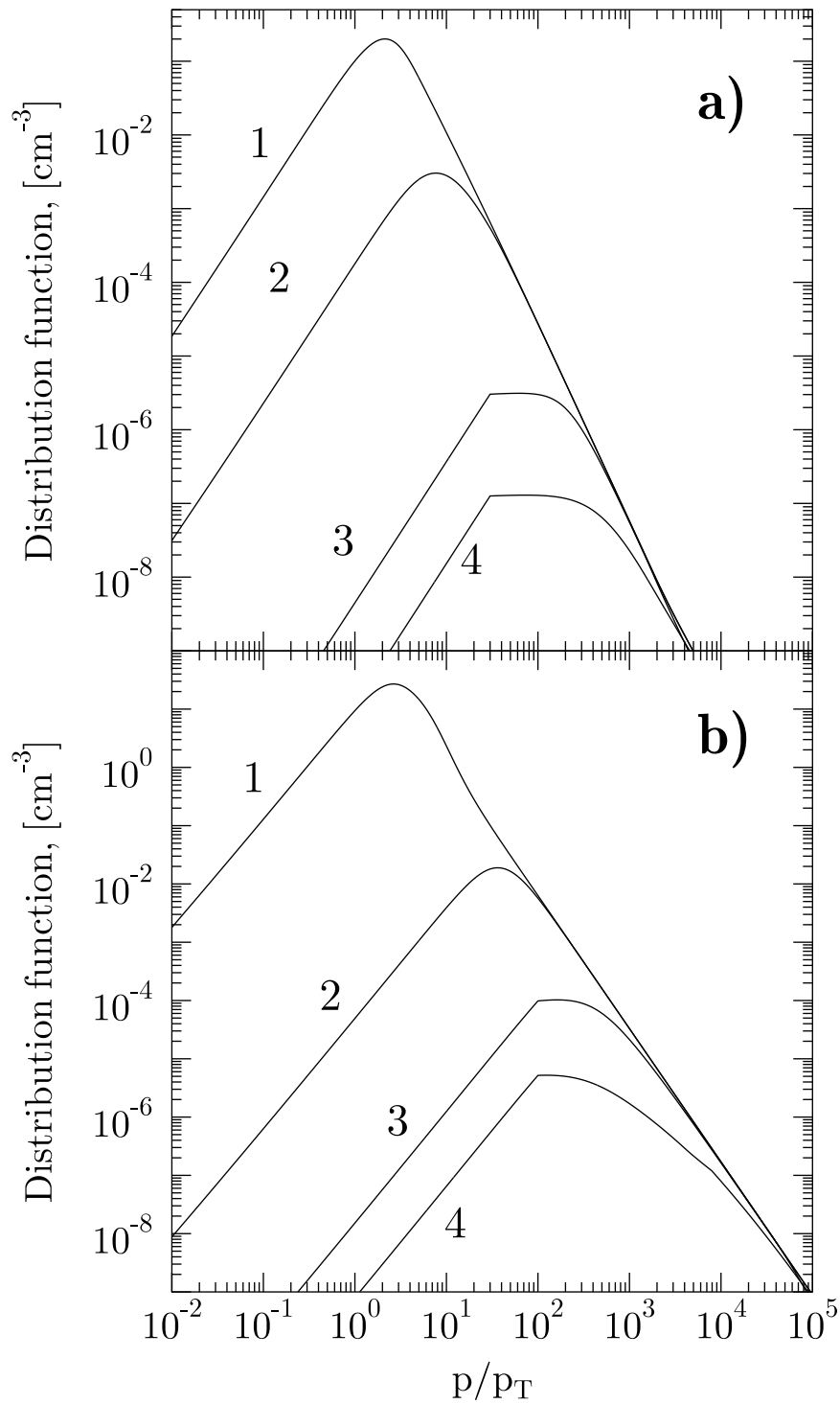

Fig. 1. The nonthermal electron distribution function $(\tilde{N}(p, x)=$ $\left.N(p, x) p^{2} p_{\mathrm{T}}\right)$ as a function of dimensionless particle momentum $p / p_{\mathrm{T}}$ ( $p_{\mathrm{T}}$ is the momentum of a thermal electron in the upstream region). We show the distributions at different distances from the bow shock plane for different ambient media: a) a fragment of velocity $v_{\mathrm{k}}=$ $3200 \mathrm{~km} \mathrm{~s}^{-1}$ in a tenuous medium; b) an SN fragment of velocity $v_{\mathrm{k}}=1080 \mathrm{~km} \mathrm{~s}^{-1}$ in a molecular cloud (see Sect. 2.3 for details). The depths from the bow shock plane are $10^{6}, 10^{12}, 10^{15}, 3 \times 10^{16} \mathrm{~cm}$ for the curves $1-4$, respectively.

gas electron temperatures are different for the two ambient media we displayed in Fig. 1. In Fig. 1 we showed only nonthermal parts of the electron distributions because the electron temperatures in both presented cases are below those required for efficient $\mathrm{K}_{\alpha}$ line production in the ejecta fragments. The threshold for $\mathrm{K}$-shell ionization of $\mathrm{Si}$ is $1.8 \mathrm{keV}$ (i.e. $p / p_{\mathrm{T}}>$ 33 in Fig. 1) while that for $\mathrm{Fe}$ is about $7.1 \mathrm{keV}$ (i.e. $p / p_{\mathrm{T}}>$ 64 in Fig. 1). Fast electrons accelerated in the MHD collisionless shock diffuse through the postshock layer and cold metallic knot suffering Coulomb losses as is clear from the Fig. 1. They produce the $\mathrm{K}_{\alpha}$ lines in the fragment body due to radiative transitions following the removal of the $1 \mathrm{~s}$ atomic electrons.

\subsection{K-shell ionization and X-ray line emissivity}

To calculate the production rate of characteristic X-ray line emission from the metal rich fragment irradiated by an intense flux of energetic electrons accelerated by an MHD shock wave the atomic inner shell ionization cross sections and the fluorescent yields are required. The experimental electron-impact $\mathrm{K}$-shell ionization cross-sections and the fluorescent yields were compiled recently by Liu et al. (2000). The standard Bethe theory of inner shell ionization (e.g. Powell 1976) was used to fit the data for nonrelativistic energies of ionizing electrons, from the threshold up to $50 \mathrm{keV}$ and the relativistic formulae by Scofield (1978) above $50 \mathrm{keV}$.

We fit the K-shell ionization cross section following the parameterization given by Scofield (1978)

$\sigma_{i}=\frac{A}{\beta^{2}} \cdot\left(b+b_{1}+b_{2} / \epsilon+b_{3} / p^{2}+b_{4} \cdot b / p+b_{5} / p^{4}\right)$.

Here the cross-section $\sigma_{i}$ is given as a function of the incident electron momentum $p$ (measured in $\left.m_{\mathrm{e}} c\right), b=\ln \left(\mathrm{p}^{2}\right)-\beta^{2}$, $\beta=v / c$. The fitting parameters $A, b_{1}-b_{5}$ were calculated by Scofield (1978) for the ions of $Z \geq 18$. We corrected the fitting parameters to account for the present laboratory measurements compiled by Liu et al. (2000).

The logarithmic asymptotic of the crossection at relativistic energies, given by Eq. (6) is important for our modeling. One can see in Fig. 1 (curves 3 and 4 ) that the electron spectra have rather flat maxima at mildly relativistic energies $\left(p / p_{\mathrm{T}} \gtrsim 400\right)$ in the most of the volume of a dense metal-rich fragment. Thus, the K-shell ionizations by relativistic electrons are substantial.

The radiative decay of $\mathrm{K}$-shell vacancy induced by fast electron results in $\mathrm{K}_{\alpha}(2 \mathrm{p} \rightarrow 1 \mathrm{~s})$ and $\mathrm{K}_{\beta}(3 \mathrm{p} \rightarrow 1 \mathrm{~s})$ lines production. The $I\left(\mathrm{~K}_{\beta}\right) / I\left(\mathrm{~K}_{\alpha}\right)$ intensity ratio is about 0.14 (e.g. Scofield 1974) for FeI-FeIX and it is decreasing for higher iron ionization stages (e.g. Jakobs \& Rozsnyai 1986). The ratio is an increasing function of the atomic number $Z$. Note however, that the optical depths for $\mathrm{K}_{\beta}$ and $\mathrm{K}_{\alpha}$ lines could be different, especially for highly ionized iron, and that would affect the observed line ratio. Thus, observing the ratio $I\left(\mathrm{~K}_{\beta}\right) / I\left(\mathrm{~K}_{\alpha}\right)$ one may constrain the source optical depth. Both $I\left(\mathrm{~K}_{\alpha}\right)$ and $I\left(\mathrm{~K}_{\beta}\right)$ Fe lines were detected recently with Chandra from Sgr B2 giant molecular cloud by Murakami et al. (2001). The observed ratio is somewhat below 0.2 indicating some possible optical depth effect, but it is still marginally consistent with the value 0.14 expected for a transparent system.

It is important to note here that shock-accelerated energetic nuclei can also provide efficient K-shell ionization. The emission spectra from the decay of inner-shell ionizations produced by a collision with an MeV-regime ion have multiple satellites (peaks) at energies higher than that of the electron-induced $\mathrm{K}_{\alpha}$ line (e.g. Garcia et al. 1973). We shall discuss the effect of ions in detail elsewhere.

\subsection{The optical depth effect}

The maximal column density of a metal (of atomic weight $A$ ) in a spherical ejecta fragment $N_{\max }^{(A)}=5.6 \times$ $10^{22}\left(M^{(A)} / M_{\odot}\right) A^{-1} R_{17}^{-2} \mathrm{~cm}^{-2}$ can be high enough to provide 
optical depths $\tau \gtrsim 1$. We use below $N^{(A)}=(1 / 2) N_{\max }^{(A)}$ as the resonant scattering column density in the model assuming the shock situated at the fragment center. The resonant line scattering effect (see, for the review, Mewe 1990) can be important for the metal rich SN fragments. The optical depth at the line center of an ion due to the resonant line scattering was given by Kaastra \& Mewe (1995) and can be expressed as:

$\tau \approx 1.34 f_{\mathrm{abs}} N_{15}^{(A)} E_{\mathrm{ph}}^{-1}(A / T)^{1 / 2}\left(1+0.5 w_{6}^{2} A / T\right)^{-1 / 2}$.

where $E_{\mathrm{ph}}$ (in $\mathrm{keV}$ ) is the line photon energy, $f_{\mathrm{abs}}$ is the absorption oscillator strength, $T$ is the ion temperature (in eV), $N_{15}^{(A)}$ is the ion column density (in $10^{15} \mathrm{~cm}^{-2}$ ), $w_{6}$ is the gas microturbulence velocity (in $10 \mathrm{~km} \mathrm{~s}^{-1}$ ).

To describe the radiative transfer for the line radiation produced inside the absorbing medium we used a simplified escape probability approach. The mean escape probability $p_{\mathrm{f}}(\tau)$ can be approximated as $p_{\mathrm{f}}(\tau) \approx[1+a \tau]^{-1}$, where $a \approx 0.43$ for $\tau \lesssim 50$ (Kaastra \& Mewe 1995). The photon absorption due to the resonant scattering of the K-shell line is most important for the ions with an incomplete L-shell (e.g. OI, Si VI, Ar X, Fe XVIII) and relatively low fluorescent yields. The resonant absorption is not effective for the neutral or the low ionization stages of $\mathrm{Fe}, \mathrm{Ca}, \mathrm{Ar}, \mathrm{Si}$ with completed L-shells where we applied the photoabsorption by Morrison \& McCammon (1983).

\section{X-ray emission from an $\mathrm{SN}$ fragment interacting with a molecular cloud}

Core-collapsed SNe from massive progenitors are expected to be correlated with massive molecular clouds. The most spectacular manifestations of the phenomena are the SNRs in starburst galaxies like NGC 253, Arp 220, M82 etc. (see e.g. Chevalier \& Fransson 2001). There are also the star-forming molecular clouds Sgr A, Sgr B2 in the Galactic Center region with a strong CS emission from dense molecular gas (e.g. Blitz et al. 1993). To model such a case we considered an SN fragment of radius $3 \times 10^{16} \mathrm{~cm}$ and of oxygen mass of $10^{-3} M_{\odot}$, containing also $\sim 10^{-4} M_{\odot}$ of an impurity (Fe, Ar, Si). The fragment is propagating through a molecular clump of number density $10^{3} \mathrm{~cm}^{-3}$ with a velocity $\gtrsim 1000 \mathrm{~km} \mathrm{~s}^{-1}$. The magnetic field value in the cloud is about $100 \mu \mathrm{G}$ and $k_{0} \approx 10^{18} \mathrm{~cm}^{2} \mathrm{~s}^{-1}$ at $1 \mathrm{keV}$.

We calculated the local emissivities of K-shell lines through the fragment depth as well as the integrated line luminosities. Table 1 contains the luminosities of $\mathrm{K}_{\alpha}$ lines of $\mathrm{O}, \mathrm{Si}, \mathrm{Ar}, \mathrm{Fe}$. These luminosities are not corrected for the optical depth effect. We have also given the maximal depths $\tau_{\max }$ for resonant scattering calculated on the assumption that the column density of a given charge state of the ion $\mathrm{A}$ is equal to $N_{\max }^{(A)}$. To obtain a real estimation one should correct the depth for the actual column densities of the ions with incomplete L-shells. Note that in Tables 1 and 2 we presented only the integral luminosities of the $\mathrm{K}_{\alpha}$ complex. A prediction for the line shape depends on the details of the ion charge state profile inside the fragment. The exact modeling of the ionization profile is beyond the scope of the present paper. Nevertheless, one could see that in the case when the bow shock is radiative (see below) the
Table 1. K-shell line luminosities of the fragment interacting with a molecular cloud.

\begin{tabular}{|c|c|c|c|c|}
\hline \multirow[t]{2}{*}{ Line $^{a}$} & \multicolumn{3}{|c|}{$v_{\mathrm{k}}\left(\mathrm{kms}^{-1}\right)$} & \multirow[t]{2}{*}{$\tau_{\max }$} \\
\hline & 1080 & 1620 & 2700 & \\
\hline $\mathrm{O}(0.54 \mathrm{keV})$ & 40.4 & 104 & 1638 & 33880 \\
\hline $\mathrm{Si}(1.7 \mathrm{keV})$ & 1.3 & 10 & 48 & $592^{b}$ \\
\hline $\operatorname{Ar}(2.9 \mathrm{keV})$ & 0.5 & 4 & 20 & $272^{b}$ \\
\hline $\mathrm{Fe}(6.4 \mathrm{keV})$ & 0.4 & 3 & 15 & $78^{b}$ \\
\hline$T^{(2)}\left[10^{7} \mathrm{~K}\right]$ & 0.3 & 0.5 & 1.0 & \\
\hline
\end{tabular}

${ }^{a}$ The luminosities are in $10^{38} \mathrm{ph} \mathrm{s}^{-1}$.

${ }^{b}$ The absorption depths can be applied only for the ionization states Si VI, Ar X, Fe XVIII and higher.

dominant iron charge state in the fragment becomes lower than Fe XVIII, providing the low resonant absorption depth and the line centroid to be close to $6.4 \mathrm{keV}$. We assumed in Table 1 that the gas micro-turbulence velocity $w_{6}=1$, and the ion temperature $T \lesssim 10^{4} \mathrm{~K}$ in the fragment body. The ion temperatures $T^{(2)}$ presented in Tables 1 and 2 were calculated for the position just after the viscous subshock. The observable electron temperatures in the postshock relaxation region are somewhat lower.

As seen in Table 1, an increase in the fragment velocity results in a strong enhancement of the line luminosities. For the lower knot velocities the line emission drops down drastically because the Coulomb losses are dominating in that case. Note however that if the value of $k_{0}$ was less than $10^{18} \mathrm{~cm}^{2} \mathrm{~s}^{-1}$ (at $1 \mathrm{keV}$ ) even the fragments of lower velocity $\left(v_{\mathrm{k}}<1000 \mathrm{~km} \mathrm{~s}^{-1}\right)$ could provide a substantial line luminosity. A fast fragment interacting with CS-emitting gas of density $\gtrsim 10^{4} \mathrm{~cm}^{-3}$ could reach even higher line luminosity if $k_{0} \sim 10^{17} \mathrm{~cm}^{2} \mathrm{~s}^{-1}$ (at $1 \mathrm{keV}$ ). Since the larger fragments (for a given knot mass) are more transparent for the K-shell lines of oxygen (and other elements) a fast $\mathrm{SN}$ fragment of $R \gtrsim 3 \times 10^{17} \mathrm{~cm}$, propagating through the inter-clump medium of density $\gtrsim 10 \mathrm{~cm}^{-3}$, might have a prominent oxygen K-line.

A fast fragment of a larger scale $\gtrsim 10^{17} \mathrm{~cm}$, of the same mass $10^{-3} M_{\odot}$, entering the molecular clump will have $\rho_{\mathrm{a}} / \rho_{\mathrm{k}} \gtrsim$ 1 and would drive a strong shock into the metal-rich fragment. Such a fragment should be a source of gamma-ray lines and also light (and other spallogenic origin) elements produced by accelerated ion interactions with the metal-rich knot. It would appear as a bright transient source.

\section{X-ray emission from an $\mathrm{SN}$ fragment in a low density medium}

Many of the isolated SN fragments are propagating through a low-density hot environment. That concerns both relatively low velocity fragments moving inside the forward shock radius of $\mathrm{SN}$ and fast velocity fragments of an SN exploding in a lowdensity environment of number density $n \approx 0.1 \mathrm{~cm}^{-3}$, gas temperature $T \approx 2 \times 10^{4} \mathrm{~K}$ and magnetic field value $\sim 3 \mu \mathrm{G}$. We first considered the same $\mathrm{SN}$ fragment as that in the dense medium, but in a wider velocity range $1000<v_{\mathrm{k}}<7000 \mathrm{~km} \mathrm{~s}^{-1}$ 
Table 2. K-shell line from the fragment interacting with low-density gas.

\begin{tabular}{ccccc}
\hline \hline Line $^{a}$ & \multicolumn{3}{c}{$v_{\mathrm{k}}\left(\mathrm{km} \mathrm{s}^{-1}\right)$} & \\
& 1600 & 3200 & 6400 & \\
\hline $\mathrm{O}(0.5-0.6 \mathrm{keV})$ & 38.0 & 66.5 & 99.8 & 33880 \\
\hline $\mathrm{Si}(1.7-1.8 \mathrm{keV})$ & 2.6 & 4.5 & 6.7 & $592^{b}$ \\
\hline $\mathrm{Ar}(2.9-3.1 \mathrm{keV})$ & 1.9 & 3.4 & 5.0 & $272^{b}$ \\
\hline $\mathrm{Fe}(6.4-6.9 \mathrm{keV})$ & 0.8 & 1.4 & 2.1 & $78^{b}$ \\
\hline$T^{(2)}\left[10^{7} \mathrm{~K}\right]$ & 1.2 & 2.8 & 6.6 & \\
\hline
\end{tabular}

${ }^{a}$ The luminosities are in $10^{36} \mathrm{ph} \mathrm{s}^{-1}$.

${ }^{b}$ The absorption depths can be applied only for the ionization states Si VI, Ar X, Fe XVIII and higher.

because even very high velocity fragments are long-lived in a low density environment. The diffusion coefficient normalization at $1 \mathrm{keV}$ was fixed to be $k_{0} \approx 3 \times 10^{19} \mathrm{~cm}^{2} \mathrm{~s}^{-1}$. We summarize the simulated $\mathrm{X}$-ray line luminosities (measured in $10^{36}$ photon $^{-1}$ ) in Table 2 . One can see that the X-ray line luminosities $L_{\mathrm{x}}<10^{29} \mathrm{erg} \mathrm{s}^{-1}$ (per $10^{-4} M_{\odot}$ of Si, S, Ar, Ca, $\mathrm{Fe}$ ) are predicted from an individual $\mathrm{SN}$ fragment of the scale $3 \times 10^{16} \mathrm{~cm}$ in a tenuous medium. In Table 2 we assumed that the gas micro-turbulence velocity $w_{6}=1$, and the ion temperature $T \lesssim 10^{4} \mathrm{~K}$ in the fragment body.

However, simulations of somewhat larger $\gtrsim 10^{17} \mathrm{~cm}$ fragments show that the luminosity corrected for absorption increases to $L_{\mathrm{x}} \gtrsim 10^{30} \mathrm{erg} \mathrm{s}^{-1}$ and even higher due to decreasing of the optical depth, and Coulomb losses. It is important that the large scale fragments are much thinner (and hotter), providing a substantial amount of ions in high ionization states. These faint transparent fragments would contribute substantially to the observed diffuse X-ray line emission of highly ionized matter. Note that the fragment deceleration time is $\propto M n_{\mathrm{a}}^{-1} R^{-2}$. That implies that the fragments in the old remnants could only be observed if they spent most of the time in the tenuous medium.

\subsection{A model of the shrapnel $A$ in the Vela SNR}

We simulated the line emission from a fragment of a scale $\sim 10^{18} \mathrm{~cm}$ to model the Vela shrapnel A discovered by Aschenbach et al. (1995) and recently studied with Chandra by Miyata et al. (2001). The oxygen-dominated fragment of mass $M \sim 10^{-2} M_{\odot}$ and velocity $v_{\mathrm{k}} \gtrsim 10^{8} \mathrm{~cm} \mathrm{~s}^{-1}$ would have a deceleration time about 10000 years in an ambient medium of $n \approx 0.1 \mathrm{~cm}^{-3}$. We found that the temperature behind the fragment bow shock dominated by nonthermal particles is about $0.5 \mathrm{keV}$. The silicon line at $1.8 \mathrm{keV}$ would have a luminosity $L_{\mathrm{x}} \sim 10^{30} \mathrm{erg} \mathrm{s}^{-1}$ if $\sim 10^{-3} \mathrm{M}_{\odot}$ of $\mathrm{Si}$ is contained in the fragment and the oxygen line at $0.6 \mathrm{keV}-L_{\mathrm{x}} \gtrsim$ $10^{31} \mathrm{erg} \mathrm{s}^{-1}$. The resonant absorption depth of the Si K-shell line is $\tau_{\max } \sim 0.5$ while that of oxygen is $\tau_{\max } \sim 30$ assuming the gas micro-turbulence velocity $w_{6}=10$, and the ion temperature $T \gtrsim 10^{6} \mathrm{~K}$ in the large diluted fragment. The mean escape probabilities $p_{\mathrm{f}}(\tau)$ are about 0.8 for $\mathrm{Si}$ and 0.07 for oxygen. The optical depth effect could account for the apparent $\mathrm{Si}$ overabundance observed by Miyata et al. (2001).

\section{Discussion}

There is observational evidence for the clumpy structure of SN ejecta in the remnants of different types and ages. Observations are available for the core-collapsed SNRs (e.g. Cas A) and also for the remnants thought to be of Type Ia (see e.g. the recent XMM-Newton observations of Tycho by Decourchelle et al. 2001). Some of the SNRs are young (e.g. Cas A) and others are older (e.g. the Vela SNR).

The observation of the ejecta clumps composition would provide a valuable test to study the details of SN phenomena. The simulations of SN nucleosynthesis (e.g. Woosley \& Weaver 1995; Thielemann et al. 1996) predict the production of $\sim 0.1 M_{\odot}$ of ${ }^{56} \mathrm{Ni}$ and ${ }^{28} \mathrm{Si}$ and $\gtrsim 0.003 M_{\odot}$ of ${ }^{36} \mathrm{Ar}$ in a core-collapsed SN. The metal distribution through the ejecta at the postexplosion stage is still poorly known. There is conclusive evidence for large-scale macroscopical mixing of $\mathrm{Ni}$ in the SN 1987A ejecta (e.g. McCray 1993). However the modeling of the ${ }^{56} \mathrm{Fe}$ distribution through the ejecta at the microscopic level is rather a complicated task. This is because of the energy deposition effect from ${ }^{56} \mathrm{Ni}$ decay (see e.g. Wang \& Chevalier 2001; Blondin et al. 2001 for recent discussions). The X-ray line emission in our model is less sensitive to the details of the element distribution in a fragment because of the high penetrating ability of energetic electrons responsible for the line excitation. Even if the Fe (or other element) atoms are locked in the dust grains we would still have $\mathrm{X}$-ray line emission excited by fast electrons.

We show that the X-ray line luminosities and spectra are different for the SN ejecta fragments in a dense molecular cloud and for that in a tenuous medium. The efficiency of $\mathrm{X}$-ray line production is higher in the fragments of lower ionization state. Strong MHD shocks could transform a sizable fraction of the kinetic energy into nonthermal particles thus reducing the heating of the shocked gas and increasing its compression. The column density of the shocked gas could reach $\sim 10^{21} \mathrm{~cm}^{-2}$ and the ion temperature just behind the shock transition region is below $5 \times 10^{6} \mathrm{~K}$. Thus, the fast fragments (of velocity $v_{\mathrm{k}} \lesssim 1500 \mathrm{~km} \mathrm{~s}^{-1}$ ) moving through molecular clouds of density $>10^{3} \mathrm{~cm}^{-3}$ would drive a radiative bow shock wave. Hollenbach \& McKee (1989) have modeled the spectra of the radiative shocks. They found rich spectra of $\mathrm{H}_{2}$ emission as well as atomic fine-structure lines with strong [OI] $(63 \mu \mathrm{m})$ [OIII], [Fe II], [C II] $(158 \mu \mathrm{m})$, [Si II] $(35 \mu \mathrm{m})$, [Ne II] $(12.8 \mu \mathrm{m})$ lines. This implies a correlation of IR and optical emission of the fragment with the X-ray line emission dominated by the lines of relatively low ion charge states. The soft thermal X-ray continuum emission from the postshock gas of $T \lesssim 10^{7} \mathrm{~K}$ is not expected to be observable in that case. On the other hand nonthermal bremsstrahlung emission with the hard spectrum of a typical photon indexes $<1.5$ is predicted in our model. The continuum bremsstrahlung emission is rather sensitive to the ionization state of the fragment. Our simulations show that the X-ray line luminosity is $\lesssim 10^{-4}$ of the kinetic energy dissipation rate in the fragment's bow shock.

The lifetime of the X-ray-line-emitting fragments in a dense molecular cloud is expected to be a few times less than the fragment deceleration time. Such a fragment should appear 
as a nonthermal source showing variability on a timescale of years. The variability is most important for radio emission. Synchrotron radio emission at a level about $100 \mathrm{mJy} \operatorname{arcsec}^{-2}$ at $100 \mathrm{MHz}$ could be expected from the fragments interacting with a dense molecular cloud if the ambient magnetic field is about $0.1 \mathrm{mG}$. Radio emission from a fragment propagating through a tenuous medium is much fainter. However, MHD type instabilities may greatly enhance the magnetic fields along the fragment boundary providing substantial radio emission, see Anderson et al. (1994); Jones et al. (1996).

The X-ray line luminosity $L_{\mathrm{x}} \sim 10^{31} \mathrm{erg} \mathrm{s}^{-1}$ (per $10^{-4} M_{\odot}$ of $\mathrm{Si}, \mathrm{S}, \mathrm{Ar}, \mathrm{Ca}, \mathrm{Fe}$ ) is predicted from an individual SN fragment in a molecular cloud. It could be detected from a few kpc distance with the current detectors aboard Chandra and XMM-Newton. An obvious candidate to study is IC 443: an SNR interacting with a molecular cloud. The recent XMM-Newton observation of IC 443 by Bocchino \& Bykov (2001) has resolved the hard X-ray source 1SAX J0618.0+2227 correlated with a molecular cloud into two sources one of which is generally consistent with the ejecta fragment interpretation. A dedicated observation of 1SAX J0618.0+2227 is desirable to check the interpretation.

An ensemble of unresolved SN fragments could contribute substantially to the observed diffuse iron line emission. Diffuse iron line emission has been found by ASCA from the Galactic Centre region on a scale of about a degree along the galactic plane (Koyama et al. 1996) and from a more extended region of the Galactic Ridge (e.g. Tanaka 2002). He-like and H-like Fe-K lines analyzed by Tanaka (2002) are significantly broadened corresponding to a velocity dispersion of a few thousand $\mathrm{km} \mathrm{s}^{-1}$. The high dispersion can be naturally explained if the lines are due to unresolved faint $\mathrm{SN}$ fragments. Alternatively, Tanaka (2002) has suggested that the high dispersion is due to charge-exchange processes of low-energy cosmic rays providing the high velocities of the line emitting ions.

A molecular cloud irradiated by a hard X-ray source is expected to be observable in fluorescent X-ray lines as X-ray reflection nebulae - a new class of X-ray sources (e.g. Sunyaev et al. 1993; Sunyaev \& Churazov 1998). The observed iron line emission from the Sgr B2 (Murakami et al. 2001) and Sgr C (Murakami et al. 2001a) complexes was considered by the authors as the $\mathrm{X}$-ray reflection nebulae irradiated by a bright source in the GC which was active in the past. Clumps of X-ray line emission of neutral and highly ionized iron of equivalent widths $\sim \mathrm{keV}$ and absorption corrected $2-10 \mathrm{keV}$ fluxes $\sim 10^{-12} \mathrm{erg} \mathrm{s}^{-1} \mathrm{~cm}^{-2}$ were found by Chandra in the Galactic center region (Bamba et al. 2002). The photon indices of 2-10 keV continuum emission - while not too constrained are broadly consistent with the photon indices $\leq 1.5$ predicted by the model of SN fragments emission considered above. If a substantial fraction of the iron ejected by an SN in a molecular cloud would reside in the fast moving fragments we could expect an iron line luminosity of $L_{\mathrm{x}} \sim 10^{34} \mathrm{erg} \mathrm{s}^{-1}$ per SN which is consistent with that observed from $\mathrm{Sgr} \mathrm{C}$ complex. The line emission from the Sgr B2 is somewhat brighter and would require contributions from more than one SNe. Infrared line observations from radiative shocks that accompany $\mathrm{SN}$ fragments in molecular clouds would help to distinguish the $\mathrm{SN}$ fragments contributions. A correlation between $\mathrm{SiO}(J=2 \rightarrow 0)$ emission morphology and $6.4 \mathrm{keV} \mathrm{Fe}$ line emission on the Galactic Center large scale and also within Sgr A and B regions found recently by Martin-Pintado et al. (2000) could be consistent with the multiple SN fragments contribution. ISO observations of 18 molecular clouds in the Galactic Center region were performed by Martin-Pintado et al. (2000a). Towards most of the clouds they have detected the "ionized bubbles" with the fine structure line emission of ionized species: SIII, NeII, and in some cases also NeIII, NII, NIII, OIII. The "bubbles" could be relevant to the fragment-cloud interactions.

Nonthermal hard X-ray continuum emission from the Galactic ridge requires a population of accelerated electrons (e.g. Valinia et al. 2000; Dogiel et al. 2002). Hard continuum of the SN fragments could contribute to the observed emission. The galactic diffuse emission mapping with forthcoming INTEGRAL mission will address the issue.

Acknowledgements. I thank the referee for very constructive comments. The work was supported by INTAS-ESA 99-1627 grant.

\section{References}

Anderson, M. C., Jones, T. W., Rudnick, L., Tregillis, I. L., \& Kang, H. 1994, ApJ, L31

Aschenbach, B., Egger, R., \& Trümper, J. 1995, Nature, 373, 585

Bamba, A., Murakami, H., Senda, A., et al. 2002

[astro-ph/0202010]

Bell, A. R. 1977, MNRAS, 179, 573

Berezhko, E. G., \& Ellison, D. C. 1999, ApJ, 526, 385

Blandford, R., \& Eichler, D. 1987, Phys. Rep., 154, 2

Bleeker, J. A. M., Willingale, R., van der Heyden, K., et al. 2001, A\&A, 365, L225

Blitz, L., Binney, J., Bally, J., Lo, K. Y. \& Ho, P. T. 1993, Nature, 361, 417

Blondin, J. M., Borkowski, K. J., \& Reynolds, S. P. 2001, ApJ, 557, 782

Bocchino, F., \& Bykov, A. M. 2001, A\&A, 376, 248

Borkowski, K. J., \& Shull, J. M. 1990, ApJ, 348, 169

Bykov, A. M., \& Uvarov, Yu. A. 1999, JETP, 88, 465

Bykov, A. M., Chevalier, R. A., Ellison, D. C., \& Uvarov, Yu. A. 2000, ApJ, 538, 203

Chevalier, R. A. 1975, ApJ, 200, 698

Chevalier, R. A., \& Fransson, C. 2001, ApJ, 558, L27

Chevalier, R. A., \& Kirshner, R. P. 1979, ApJ, 233, 154

Decourchelle, A., Sauvageot, J. L., Audard, M., et al. 2001, A\&A, $365, \mathrm{~L} 218$

Drury, L. O’. C., Duffy, P., \& Kirk, J. G. 1996, A\&A, 309, 1002

Dogiel, V. A., Schönfelder, V., \& Strong, A. 2002, A\&A, 382, 730

Dorfi, E. A. 2000, Ap\&SS, 272, 227

Fesen, R. A., Morse, J. A., Chevalier, R. A., et al. 2002, AJ, 122, 2644

Garcia, J. D., Fortner, R. J., \& Kavanagh, T. M. 1973, Rev. Mod. Phys., 45,111

Hollenbach, D., \& McKee, C. F. 1989, ApJ, 342, 306

Hughes, J. P., Rakowski, C. E., Burrows, D. N., \& Slane, P. 2000, ApJ, 528, L109

Hwang, U., Szymkowiak, A. E., Petre, R., \& Holt, S. S. 2001, ApJ, 560, L175

Jakobs, V., \& Rozsnyai, B. 1986, Phys. Rev. A, 34, 216

Jones, F. C., \& Ellison, D. C. 1991, Space Sci. Rev., 58, 259

Jones, T. W., Ryu, D., \& Tregillis, I. L. 1996, ApJ, 473, 365

Kaastra, J. S., \& Mewe, R. 1995, A\&A, 302, L13 
Koyama, K., Maeda, Y., Sonobe, T., et al. 1996, PASJ, 48, 249

Laming, J. M. 2001, ApJ, 563, 828

Levinson, A. 1996, MNRAS, 278, 1018

Liu, M., An, Z., Tang, C., et al. 2000, ADNDT, 76, 213

Malkov, M. A., \& Drury, L. O'. C. 2001, Rep. Progr. Phys., 64, 429

Martin-Pintado, J., de Vicente, P., Rodriguez-Fernandez, N. J., Fuente, A., \& Planesas, P. 2000, A\&A, 356, L5

Martin-Pintado, J., Rodriguez-Fernandez, N. J., de Vicente, P., et al. 2000a, in ISO beyond the peaks, ed. A. Salama et al., ESA-SP 456, 111

McCray, R. 1993, ARA\&A, 31, 175

Mewe, R. 1990, in Physical Processes in Hot Cosmic Plasmas, ed. W. Brinkmann et al. (Kluwer), 39

Miyata, E., Tsunemi, H., Aschenbach, B., \& Mori, K. 2001, ApJ, 559, L45

Morisson, D., \& McCammon, D. 1983, ApJ, 270, 119

Murakami, H., Koyama, K., \& Maeda, Y. 2001, ApJ, 558, 687

Murakami, H., Koyama, K., Tsujimoto, M., Maeda, Y., \& Sakano, M. 2001a, ApJ, 550, 297

Park, S., Roming, P. W. A., Hughes, J. P., et al. 2001, ApJ, 564, L39

Porquet, D., Arnaud, M., \& Decourchelle, A. 2001, A\&A, 373, 1110

Powell, C. J. 1976, Rev. Mod. Phys., 48, 33

Raymond, J. C. 1984, ARA\&A, 22, 75
Raymond, J. C. 2001, Space Sci. Rev., 99, 209

Scofield, J. H. 1974, Phys. Rev. A, 9, 1041

Scofield, J. H. 1978, Phys. Rev. A, 18, 963

Shimada, N., Terasawa, T., Hoshino, M., et al. 1999, Ap\&SS, 264, 481

Shull, J. M., \& McKee, C. F. 1979, ApJ, 227, 131

Sunyaev, R. A., \& Churazov, E. 1998, MNRAS, 297, 1279

Sunyaev, R. A., Markevitch, M., \& Pavlinsky, M. 1993, ApJ, 407, 606

Sutherland, R. S., \& Dopita, M. A. 1995, ApJ, 439, 381

Tanaka, Y. 2002, A\&A, 382, 1052

Tanaka, Y., Miyaji, T., \& Hasinger, G. 1999, Astron. Nachr., 320, 181

Terasawa, T., Maezawa, K., Hoshino, M., et al. 1999, in Proc. 26th Int. Cosmic Ray Conf. (Salt Lake City) 6, 528

Thielemann, F.-K., Nomoto, K., \& Hashimoto, M.-A. 1996, ApJ, 460, 408

Valinia, A., Tatischeff, V., Arnaud, K., et al. 2000, ApJ, 543, 733

Vink, J., Kaastra, J., \& Bleeker, J. 1997, A\&A 328, 628

Wang, C-Y., \& Chevalier, R. A. 2001, ApJ, 549, 1119

Wang, Q. D., Gotthelf, E. V., \& Lang, C. C. 2002, Nature, 415, 148

Willingale, R., Bleeker, J. A. M., van der Heyden, K., Kaastra, J. S., \& Vink, J. 2002, A\&A, 381, 1039

Winkler, P. F., \& Kirshner, R. P. 1985, ApJ, 299, 981

Woosley, S. E., \& Weaver, T. A. 1995, ApJS, 101, 181 